\title{
Secondary suturing compared to non-suturing for broken down perineal wounds following childbirth (Protocol)
}

\author{
Dudley LM, Kettle C, Ismail KMK
}

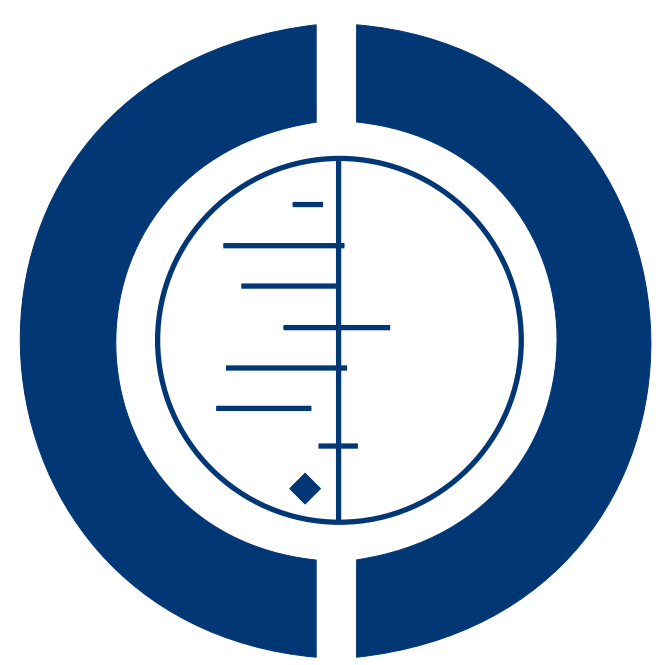

\section{THE COCHRANE COLLABORATION $^{\circledR}$}

This is a reprint of a Cochrane protocol, prepared and maintained by The Cochrane Collaboration and published in The Cochrane Library 2011, Issue 2

http://www.thecochranelibrary.com

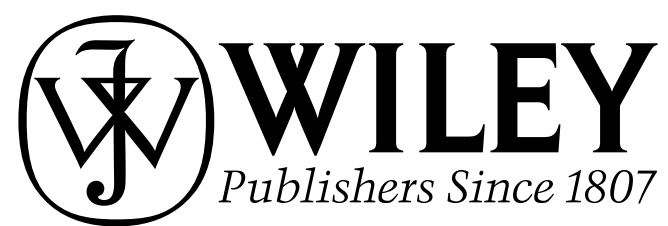

Secondary suturing compared to non-suturing for broken down perineal wounds following childbirth (Protocol)

Copyright (C) 20II The Cochrane Collaboration. Published by John Wiley \& Sons, Ltd. 
TABLE OF CONTENTS

HEADER . . . . . . . . . . . . . . . . . . . . . . . . . . . . . . . . . . . . . . . . 1

ABSTRACT . . . . . . . . . . . . . . . . . . . . . . . . . . . . . . . . . . . . . . . . . . . . . . .

BACKGROUND . . . . . . . . . . . . . . . . . . . . . . . . . . . . . . . . . . . . . . . . . . . . .

OBJECTIVES . . . . . . . . . . . . . . . . . . . . . . . . . . . . . . . . . . . . . . . . . . . . . .

METHODS . . . . . . . . . . . . . . . . . . . . . . . . . . . . . . . . . . . . . . 3

ACKNOWLEDGEMENTS . . . . . . . . . . . . . . . . . . . . . . . . . . . . . . . . . . . . . . . . . . 7

REFERENCES . . . . . . . . . . . . . . . . . . . . . . . . . . . . . . . . . . . . . 7

HISTORY . . . . . . . . . . . . . . . . . . . . . . . . . . . . . . . . . . . . . . . 8

CONTRIBUTIONS OF AUTHORS . . . . . . . . . . . . . . . . . . . . . . . . . . . . . . . . . . . . . 8

DECLARATIONS OF INTEREST . . . . . . . . . . . . . . . . . . . . . . . . . . . . . . . . . . . . . . . 9

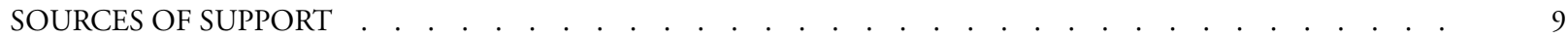


[Intervention Protocol]

\title{
Secondary suturing compared to non-suturing for broken down perineal wounds following childbirth
}

\author{
Lynn M Dudley ${ }^{1}$, Christine Kettle ${ }^{1}$, Khaled MK Ismail ${ }^{2}$ \\ ${ }^{1}$ Maternity Centre, University Hospital of North Staffordshire, Stoke-on-Trent, UK. ${ }^{2}$ Institute of Science and Technology in Medicine, \\ Keele University Medical School, Keele, UK \\ Contact address: Lynn M Dudley, Maternity Centre, University Hospital of North Staffordshire, Newcastle Road, Stoke-on-Trent, \\ Staffordshire, ST4 6QG, UK. lynn.dudley@uhns.nhs.uk.
}

Editorial group: Cochrane Pregnancy and Childbirth Group.

Publication status and date: New, published in Issue 2, 2011.

Citation: Dudley LM, Kettle C, Ismail KMK. Secondary suturing compared to non-suturing for broken down perineal wounds following childbirth. Cochrane Database of Systematic Reviews 2011, Issue 2. Art. No.: CD008977. DOI: 10.1002/14651858.CD008977.

Copyright (C) 2011 The Cochrane Collaboration. Published by John Wiley \& Sons, Ltd.

\section{A B S T R A C T}

This is the protocol for a review and there is no abstract. The objectives are as follows:

The objective for this review is to evaluate the therapeutic effectiveness of re-suturing of dehisced perineal wounds compared to nonsuturing (healing by secondary intention). 


\section{B A C K G R O U N D}

Approximately 350,000 women in the United Kingdom every year undergo perineal repair following childbirth to facilitate healing of the trauma site (Kettle 2002; McCandlish 1998; RCOG 2004). Despite the large numbers of women undergoing perineal repair and given that the postpartum management of perineal trauma, including the prevention of wound infection and assessing wound healing, are core components of routine maternity care (Gould 2007; NICE 2006; Steen 2007) there is limited research evidence available on the management and consequences of perineal wound dehiscence. It is apparent that perineal wound dehiscence (also referred to as wound breakdown) both nationally and worldwide has not been a high priority either in practice or research and therefore current management is unlikely to be based upon robust evidence. Due to the lack of evidence-based clinical guidelines, clinical practice varies widely between individual practitioners and institutions.

There are some suggestions that the early closure of dehisced perineal wounds should be attempted in order to maintain perineal integrity (ACOG 2006; Hankins 1990; Monberg 1987; Ramin 1992; Uygur 2004); however, most dehisced perineal wounds are left to heal naturally by secondary intention (expectant management). Healing by secondary intention is a process whereby the dehisced area fills with granulation tissue that gradually contracts to bring the wound edges together; however, this is a slow process and can take several weeks for the wound to completely heal (Boyle 2006; Thomas 1990).

Perineal wound dehiscence, which is commonly reported to be associated with infection (Gould 2007; Ramin 1992; Tharpe 2008), may lead to major physical, psychological and social problems if left untreated. Although maternal mortality is extremely rare in developed countries, an infected perineal wound is a potential route for systemic infection whereby sepsis and septic shock may ensue (Lewis 2007; Rotas 2007). Gallop 2002 in a retrospective case report analysis revealed the death of a young 25 -year-old mother five days postnatal, who died as a consequence of an overwhelming sepsis with a necrotising fasciitis, associated with an infected episiotomy site.

The morbidity, however, associated with perineal wound dehiscence, can and does pose a serious threat to the general well being and quality of life of the mother. This was clearly demonstrated in a case study by Rose 2005 which revealed that infection and episiotomy dehiscence with rectal injury can be a catastrophic event for the woman and may result in an ileostomy (a surgical opening constructed by bringing the end or loop of small intestine (the Ileum) out onto the surface of the skin).

The traditional approach towards the management of dehisced perineal wounds, often results in a protracted period of significant morbidity for women, which centres around persistent pain and discomfort at the perineal wound site, urinary retention, defecation problems, dyspareunia (painful sexual intercourse) and psy- chological and psychosexual issues from embarrassment and altered body image (Hankins 1990; Ramin 1992; Steen 2007; Uygur 2004; Williams 2006). The relationship with her newborn baby may become affected, whilst the ability to breastfeed may also be prevented due to the distress cause by perineal problems (Sleep 1991). Consequently this may be detrimental to the woman's relationship with her partner and other family members, which may lead to relationship or marriage breakdown. Additionally, there are extra financial costs to the women in relation to hospital visits, transport costs and even delay in returning to employment in some circumstances. Perineal wound dehiscence is also a burden on hospital resources, as quite often women who suffer this consequence of childbirth have to undergo corrective surgery, perineal refashioning, and excision of excessive scar tissue or other procedures associated with treating perineal dysfunction (Ganapathy 2008).

Perineal wound complications are feared by many pregnant and recently delivered women (Al-Mufti 1997; Bick 2010; Clements 2001). On occasions, some women are so traumatised by their experience of poor perineal management they request subsequent deliveries by caesarean section. Additionally, it is concerning that women who are pregnant for the first time are becoming increasingly worried about the consequences of perineal injury following childbirth and the associated morbidity. This may also be a contributing factor towards the increasing interest in elective caesarean section as a more 'attractive' alternative mode of delivery (Wagner 2000). Indeed, a survey conducted in 2001 reported that $31 \%$ of all women said they would prefer an elective caesarean section compared to vaginal delivery and astonishingly, $80 \%$ of these would prefer a caesarean section because of the fear of perineal damage (Clements 2001).

A recent Delphi survey (Bick 2010) of a cohort of women who previously sustained a degree of perineal trauma demonstrated that the most important outcome for women is fear of perineal wound infection and wound healing both at one week and two to four weeks postnatal. These surveys were carried out in the UK (North Staffordshire and Reading) and the collaborative study team felt that the responses were possibly related to the growing concerns relating to the escalating numbers of community and hospital acquired MRSA infection. People in the UK are aware that MRSA is an emerging problem which may present as skin and soft tissue infection or sepsis with the potential of septic shock (Lewis 2007).

The Delphi study was subsequently repeated in Brazil where it was also found that perineal wound infection and wound healing were the main concerns of the women even though MRSA is not so publicised. Hence the findings of the Delphi survey confirm that the fear of perineal wound complications is not only isolated to the UK but is a true cross-cultural fear for many women.

\section{Description of the condition}


A dehisced perineal wound following a spontaneous second-, third- or fourth-degree tear or episiotomy.

\section{Definition of a dehisced perineal wound}

Separation of sutured perineal skin, vaginal mucosa or the underlying perineal muscles.

\section{Incidence of dehisced perineal wounds}

The precise incidence rate for childbirth related perineal wound dehiscence remains unknown; figures of $0.1 \%$ to $0.2 \%$ have been reported, dependent upon the degree of the initial trauma (Ramin 1994), whilst rates of $4.6 \%$ in relation to fourth-degree tears have also been suggested (Goldaber 1993).

\section{Definition of a second-degree tear}

Second-degree injury to the perineum involving perineal muscles but not the anal sphincter (RCOG 2007).

\section{Definition of a third-degree tear}

Third-degree injury to the perineum involving the anal sphincter complex (RCOG 2007).

3a: less than $50 \%$ of external anal sphincter (EAS) thickness torn.

3b: more than $50 \%$ of EAS thickness torn.

3c: both EAS and internal anal sphincter (IAS) torn.

\section{Definition of a fourth-degree tear}

Fourth-degree injury to the perineum involving the anal sphincter complex (EAS and IAS) and anal epithelium (RCOG 2007).

\section{Definition of an episiotomy}

Episiotomy is defined as a surgical incision of the perineum made by the midwife or obstetrician to increase the diameter of the vaginal outlet to facilitate the birth of the baby (Kettle 2007).

\section{Description of the intervention}

Resuturing of the dehisced perineal wound compared to leaving the wound to heal by expectant management (secondary intention).

\section{How the intervention might work}

Traditionally the most common approach towards the management of dehisced perineal wounds has been to avoid re-suturing, thereby allowing the wound to heal by secondary intention. This approach can result in a protracted period of significant morbidity for women. There are some reports however that suggest that early repair of perineal wound dehiscence is safe, effective and avoids the prolonged period of disability and distress inherent with healing by secondary intention (Hankins 1990; Ramin 1992; Uygur 2004). Therefore, we envisage that re-suturing within the first two weeks following childbirth may result in a reduction in the perineal pain during the healing process up to six months post delivery; a reduction in dyspareunia (painful sexual intercourse); continuation of exclusive breastfeeding up to six months; and increased satisfaction with the aesthetic results of the perineal wound.

\section{Why it is important to do this review}

Perineal wound dehiscence has not been given a high priority either in practice or research and therefore management is unlikely to be based upon robust evidence. Whilst mortality from perineal wound dehiscence is extremely rare, the associated morbidity can and does pose a serious threat to general well being and mothers' quality of life.

A systematic review of the available literature is needed to evaluate the therapeutic effectiveness of re-suturing of dehisced perineal wounds compared to non-suturing (healing by secondary intention).

\section{O B J E C T IVES}

The objective for this review is to evaluate the therapeutic effectiveness of re-suturing of dehisced perineal wounds compared to non-suturing (healing by secondary intention).

\section{METHODS}

\section{Criteria for considering studies for this review}

\section{Types of studies}

- We will include all published and unpublished randomised controlled trials investigating resuturing versus expectancy for dehisced perineal wounds (second-, third- and fourth-degree tears and episiotomy) following childbirth.

- We will exclude non-randomised, quasi-randomised, cluster-randomised, and crossover trial designs.

- We will include studies presented as abstracts if sufficient information on the study design and outcome data are available. 


\section{Types of participants}

All women with a dehisced perineal wound following primary repair of a spontaneous second-, third- or fourth-degree tear or episiotomy within the first two weeks following childbirth.

\section{Types of interventions}

Any secondary suturing of dehisced perineal wounds (second, third- or fourth-degree tear or episiotomy), following wound debridement and the removal of any remaining suture material within the first six weeks following childbirth compared with nonsuturing.

All re-sutured perineal wounds will be included irrespective of suture material.

\section{Types of outcome measures}

\section{Primary outcomes}

- Proportion of women with a healed perineal wound at 6-8 weeks

\section{Secondary outcomes}

- Pain at six weeks, three months and six months.

- Dyspareunia at three to six months.

- Women's satisfaction with the aesthetic results of the perineal wound.

- Rates of breastfeeding (at six weeks and at six months).

- Rates of exclusive breastfeeding (at six weeks and six months).

- Maternal depression.

- Maternal anxiety.

\section{Definition of wound healing}

Wound healing is defined as the physiological processes by which the body both replaces and restores function to the damaged tissues (Flanagan 1996; Tortora G 1996).

\section{Assessment of wound healing}

We will evaluate wound healing by a systematic assessment of redness, oedema, bruising, discharge and approximation of the wound edges, referred to as the REEDA scale (Davidson 1974).

\section{Search methods for identification of studies}

\section{Electronic searches}

We will contact the Trials Search Co-ordinator to search the Cochrane Pregnancy and Childbirth Group's Trials Register.
The Cochrane Pregnancy and Childbirth Group's Trials Register is maintained by the Trials Search Co-ordinator and contains trials identified from:

1. quarterly searches of the Cochrane Central Register of Controlled Trials (CENTRAL);

2. weekly searches of MEDLINE;

3. handsearches of 30 journals and the proceedings of major conferences;

4. weekly current awareness alerts for a further 44 journals plus monthly BioMed Central email alerts.

Details of the search strategies for CENTRAL and MEDLINE, the list of handsearched journals and conference proceedings, and the list of journals reviewed via the current awareness service can be found in the 'Specialized Register' section within the editorial information about the Cochrane Pregnancy and Childbirth Group.

Trials identified through the searching activities described above are each assigned to a review topic (or topics). The Trials Search Co-ordinator searches the register for each review using the topic list rather than keywords.

\section{Searching other resources}

We will search for all relevant trials in reviews, national and international guidelines and other publications identified when preparing this review.

We will not apply any language restrictions.

\section{Data collection and analysis}

\section{Selection of studies}

Three review authors (Lynn Dudley, Christine Kettle and Khaled MK Ismail) will independently assess and select trials for inclusion in this review. It will not be possible to assess the relevance of the trials blinded because we will know the authors' names, institution, journal of publication and results when we apply the inclusion criteria. We will resolve all disagreements by discussion until we reach a consensus and document reasons for exclusion.

\section{Data extraction and management}

We will design a form to extract data. For eligible studies, two review authors will extract the data using the agreed form. We will resolve discrepancies through discussion or, if required, we will consult the third review author. We will enter data into Review Manager software (RevMan 2008) and check for accuracy.

When information regarding any of the above is unclear, we will attempt to contact authors of the original reports to provide further details. 


\section{Assessment of risk of bias in included studies}

Two review authors (Lynn Dudley and Chistine Kettle) will independently assess risk of bias for each study using the criteria outlined in the Cochrane Handbook for Systematic Reviews of Interventions (Higgins 2009). We will resolve any disagreement by discussion or by involving the third review author (Khaled Ismail).

\section{(I) Sequence generation (checking for possible selection bias)}

We will describe for each included study the method used to generate the allocation sequence in sufficient detail to allow an assessment of whether it should produce comparable groups.

We will assess the method as:

- adequate (any truly random process, e.g. random number table; computer random number generator);

- inadequate (any non-random process, e.g. odd or even date of birth; hospital or clinic record number); or

- unclear.

\section{(2) Allocation concealment (checking for possible selection} bias)

We will describe for each included study the method used to conceal the allocation sequence and determine whether intervention allocation could have been foreseen in advance of, or during recruitment, or changed after assignment.

We will assess the methods as:

- adequate (e.g. telephone or central randomisation; consecutively numbered sealed opaque envelopes);

- inadequate (open random allocation; unsealed or nonopaque envelopes, alternation; date of birth);

- unclear.

\section{(3) Blinding (checking for possible performance bias)}

In view of the nature of the intervention it is not possible to blind either study participants or personnel from the intervention at the outset; however subsequent independent assessments of the primary outcome could potentially be blind to the intervention received. We will describe for each included study the methods used, if any, to blind personnel from knowledge of which intervention a participant received. We will judge studies at low risk of bias if they were blinded, or if we judge that the lack of blinding could not have affected the results. We will assess blinding separately for different outcomes or classes of outcomes.

We will assess the methods as:

- adequate, inadequate or unclear for personnel;

- adequate, inadequate or unclear for outcome assessors.
(4) Incomplete outcome data (checking for possible attrition bias through withdrawals, dropouts, protocol deviations)

We will describe for each included study, and for each outcome or class of outcomes, the completeness of data including attrition and exclusions from the analysis. We will state whether attrition and exclusions were reported, the numbers included in the analysis at each stage (compared with the total randomised participants), reasons for attrition or exclusion where reported, and whether missing data were balanced across groups or were related to outcomes. Where sufficient information is reported, or can be supplied by the trial authors, we will re-include missing data in the analyses which we undertake. We will assess methods as:

- adequate;

- inadequate:

- unclear.

We will consider excluding trials where there is more than $20 \%$ loss to follow-up.

\section{(5) Selective reporting bias}

We will describe for each included study how we investigated the possibility of selective outcome reporting bias and what we found. We will assess the methods as:

- adequate (where it is clear that all of the study's prespecified outcomes and all expected outcomes of interest to the review have been reported);

- inadequate (where not all the study's pre-specified outcomes have been reported; one or more reported primary outcomes were not pre-specified; outcomes of interest are reported incompletely and so cannot be used; study fails to include results of a key outcome that would have been expected to have been reported);

- unclear.

\section{(6) Other sources of bias}

We will describe for each included study any important concerns we have about other possible sources of bias.

We will assess whether each study was free of other problems that could put it at risk of bias:

- yes;

- no;

- unclear.

\section{(7) Overall risk of bias}

We will make explicit judgements about whether studies are at high risk of bias, according to the criteria given in the Handbook (Higgins 2009). With reference to (1) to (6) above, we will assess the likely magnitude and direction of the bias and whether we consider it is likely to impact on the findings. We will explore the impact of the level of bias through undertaking sensitivity analyses - see Sensitivity analysis. 


\section{Measures of treatment effect}

\section{Dichotomous data}

For dichotomous data, we will present results as summary risk ratio with $95 \%$ confidence intervals.

\section{Continuous data}

For continuous data, we will use the mean difference if outcomes are measured in the same way between trials. We will use the standardised mean difference to combine trials that measure the same outcome, but use different methods.

\section{Unit of analysis issues}

We will only include randomised controlled trials in which the participants are individually randomised into the clinical trials. We will not include crossover trials or cluster-randomised trials in this review.

\section{Dealing with missing data}

For included studies, we will note levels of attrition. We will explore the impact of including studies with high levels of missing data in the overall assessment of treatment effect by using sensitivity analysis.

For all outcomes, we will carry out analyses, as far as possible, on an intention-to-treat basis, i.e. we will attempt to include all participants randomised to each group in the analyses, and analyse all participants in the group to which they were allocated, regardless of whether or not they received the allocated intervention. The denominator for each outcome in each trial will be the number randomised minus any participants whose outcomes are known to be missing.

\section{Assessment of heterogeneity}

We will assess statistical heterogeneity in each meta-analysis using the $\mathrm{T}^{2}, \mathrm{I}^{2}$ and $\mathrm{Chi}^{2}$ statistics. We will regard heterogeneity as substantial if $\mathrm{I}^{2}$ is greater than $30 \%$ and either $\mathrm{T}^{2}$ is greater than zero, or there is a low $\mathrm{P}$ value (less than 0.10 ) in the $\mathrm{Chi}^{2}$ test for heterogeneity.

\section{Assessment of reporting biases}

If there are 10 or more studies in the meta-analysis we will investigate reporting biases (such as publication bias) using funnel plots. We will assess funnel plot asymmetry visually, and use formal tests for funnel plot asymmetry. For continuous outcomes we will use the test proposed by Egger 1997, and for dichotomous outcomes we will use the test proposed by Harbord 2006. If we detect asymmetry in any of these tests or by a visual assessment, we will perform exploratory analyses to investigate it.

\section{Data synthesis}

We will carry out statistical analysis using the Review Manager software (RevMan 2008). We will use fixed-effect meta-analysis for combining data where it is reasonable to assume that studies are estimating the same underlying treatment effect: i.e. where trials are examining the same intervention, and the trials' populations and methods are judged sufficiently similar. If there is clinical heterogeneity sufficient to expect that the underlying treatment effects differ between trials, or if substantial statistical heterogeneity is detected, we will use random-effects meta-analysis to produce an overall summary if an average treatment effect across trials is considered clinically meaningful. We will treat the random-effects summary as the average range of possible treatment effects and we will discuss the clinical implications of treatment effects differing between trials. If the average treatment effect is not clinically meaningful we will not combine trials.

If we use random-effects analyses, we will present the results as the average treatment effect with its $95 \%$ confidence interval, and the estimates of $\mathrm{T}^{2}$ and $\mathrm{I}^{2}$.

\section{Subgroup analysis and investigation of heterogeneity}

If we identify substantial heterogeneity, we will investigate it using subgroup analyses and sensitivity analyses. We will consider whether an overall summary is meaningful, and if it is, use random-effects analysis to produce it.

We plan to carry out the following subgroup analyses.

1. Comparison by degree of initial trauma; spontaneous second-, third-, fourth-degree perineal tears; episiotomy.

2. Spontaneous vaginal delivery versus instrumental delivery.

3. Nulliparous versus multiparous.

4. Spontaneous onset of labour versus induction of labour.

5. Comparison by methods of pain relief in labour: entonox ( $50 \%$ nitrous oxide and $50 \%$ oxygen), intramuscular opioids, epidural.

6. Singleton versus multiple pregnancies.

7. Medical conditions which may impact upon wound healing such as diabetes versus no reported medical condition.

8. Demographic data including maternal age and weight.

We will restrict subgroup analysis to the primary outcome: the proportion of women with a healed perineal wound at 6-8 weeks. For fixed-effect inverse variance meta-analyses we will assess differences between subgroups by interaction tests. For random-effects and fixed-effect meta-analyses using methods other than inverse variance, we will assess differences between subgroups by inspection of the subgroups' confidence intervals; non-overlapping confidence intervals indicate a statistically significant difference in treatment effect between the subgroups.

\section{Sensitivity analysis}

We will perform sensitivity analyses for the primary outcome where there is risk of bias associated with the quality of the in- 
cluded trials. We will aim to carry out sensitivity analysis to explore the effects of fixed-effect or random-effects analyses for outcomes with statistical heterogeneity.

\section{ACKNOWLEDGEMENTS}

As part of the pre-publication editorial process, this protocol has been commented on by three peers (an editor and two referees who are external to the editorial team) and the Group's Statistical Adviser.

\section{R E F E R E N C E S}

\section{Additional references}

\section{ACOG 2006}

American College of Obstetrics and Gynecology. ACOG Practice Bulletin No. 71: Episiotomy. Obstetrics \& Gynecology 2006;107(4):957-62.

\section{Al-Mufti 1997}

Al-Mufti R, McCarthy A, Fisk NM. Survey of obstetricians' personal preference and discretionary practice. European Journal of Obstetrics \& Gynecology and Reproductive Biology 1997;73(1):1-4.

\section{Bick 2010}

Bick D. A Delphi survey of women who have experienced perineal trauma. Personal communication 2010.

Boyle 2006

Boyle M. Wound healing in midwifery. Oxford: Radcliffe, 2006.

\section{Clements 2001}

Clements RV. Risk management and litigation in obstetrics. London: RSM press/Royal College of Obstetricians and Gynaecologists, 2001.

\section{Davidson 1974}

Davidson NS. REEDA Evaluation postpartum healing. Journal of Nurse Midwifery 1974;19:6-8.

\section{Egger 1997}

Egger M, Davey Smith G, Schneider M, Minder C. Bias in meta-analysis detected by a simple, graphical test. $B M J$ 1997;315(7109):629-34.

\section{Flanagan 1996}

Flanagan M. A practical framework for wound assessment 1 : Physiology. British Journal of Nursing 1996;5(22):1391-97.

\section{Gallop 2002}

Gallop DG, Freedman MA, Meguiar RV, Freedman $\mathrm{SN}$, Nolan TE. Necrotizing fasciitis in gynecologic and obstetric patients: a surgical emergency. American Journal of Obstetrics and Gynecology 2002;187(2):305-10.

\section{Ganapathy 2008}

Ganapathy R, Bardis NS, Lamont RF. Secondary repair of the perineum following childbirth. Journal of Obstetrics and Gynaecology 2008;28(6):608-13.

Goldaber 1993

Goldaber KG, Wendel PJ, McIntire DD, Wendel GD. Postpartum perineal morbidity after fourth-degree perineal repair. American Journal of Obstetrics and Gynaecology 1993; 168:489-93.

\section{Gould 2007}

Gould D. Perineal tears and episiotomy. Nursing Standard 2007;21(52):41-6.

Hankins 1990

Hankins GDV, Heath JC, Gilstrap LC, Hammond TL, Yeomans ER, Snyder RR. Early repair of episiotomy dehiscence. Obstetrics \& Gynecology 1990;75(48):48-51.

\section{Harbord 2006}

Harbord RM, Egger M, Sterne JA. A modified test for small-study effects in meta-analyses of controlled trials with binary endpoints. Statistics in Medicine 2006;25:3443-57.

\section{Higgins 2009}

Higgins JPT, Green S, editors. Cochrane Handbook for Systematic Reviews of Interventions Version 5.0.2 [updated September 2009]. The Cochrane Collaboration, 2009. Available from www.cochrane-handbook.org.

\section{Kettle 2002}

Kettle C, Hills RK, Jones P, Darby L, Gray R, Johanson R. Continuous versus interrupted perineal repair with standard or rapidly absorbed sutures after spontaneous vaginal birth: a randomised controlled trial. Lancet 2002;359(9325): 2217-23.

\section{Kettle 2007}

Kettle C, Hills RK, Ismail KMK. Continuous versus interrupted sutures for repair of episiotomy or second degree tear. Cochrane Database of Systematic Reviews 2007, Issue 4. [DOI: 10.1002/14651858.CD000947.pub2] 
Lewis 2007

Lewis G. Confidential enquiry into maternal and child health. Why mothers die: the Seventh Report of Confidential Enquiries into Maternal Deaths in the United Kingdom 2003-2005. London: RCOG, 2007.

\section{McCandlish 1998}

McCandlish R, Bowler U, Van Asten H, Berridge G,

Winter C, Sames L, et al.A randomised controlled trial of the care of the perineum during the second stage of labour. British Journal of Obstetrics and Gynaecology 1998;105(12): 1262-72.

\section{Monberg 1987}

Monberg J, Hammen S. Ruptured episiotomia resutured primarily. Acta Obstetricia et Gynecologica Scandinavica 1987;66(2):163-4.

\section{NICE 2006}

NICE. Routine postnatal care for women and their babies. NICE Clinical Guideline, Number 37. London: NICE, 2006.

\section{Ramin 1992}

Ramin SM, Ramus RM, Little BB, Gilstrap LC. Early repair of episiotomy dehiscence associated with infection. American Journal of Obstetrics and Gynecology 1992;167(4): 1104-7.

\section{Ramin 1994}

Ramin SM, Gilstrap LC. Episiotomy and early repair of dehiscence. Clinical Obstetrics and Gynaecology 1994;37: 816-23.

\section{RCOG 2004}

Royal College of Obstetricians and Gynaecologists. Methods and materials used in perineal repair. Clinical Guideline Number 23. London: RCOG, 2004.

\section{RCOG 2007}

Royal College of Obstetricians and Gynaecologists. The management of third and fourth degree perineal tears. Clinical Guideline Number 29. London: RCOG, 2007.

\section{RevMan 2008}

The Nordic Cochrane Centre, The Cochrane Collaboration. Review Manager (RevMan). 5.0. Copenhagen: The Nordic Cochrane Centre, The Cochrane Collaboration, 2008.

\section{Rose 2005}

Rose C, Blessitt M, Araghizadeh F, Morrison J. Episiotomy dehiscence that required intestinal diversion. American Journal of Obstetrics and Gynecology 2005;193(5):1759-60.

\section{Rotas 2007}

Rotas M, McCalla S, Liu C, Minkoff H. Methicillinresistant Staphylococcus aureus necrotizing pneumonia arising from an infected episiotomy site. Obstetrics \& Gynecology 2007;109(2 Pt 2):533-6.

\section{Sleep 1991}

Sleep J. Perineal care: a series of five randomised controlled trials. In: Robinson S, Thompson AM editor(s). Midwives research and childbirth. Vol. 2, London: Chapman and Hall, 1991:199-251.

\section{Steen 2007}

Steen M. Perineal tears and episiotomy: how do wounds heal?. British Journal of Midwifery 2007;15(5):273-80.

\section{Tharpe 2008}

Tharpe N. Post pregnancy genital tract and wound infections. Journal of Midwifery and Womens Health 2008; 53:236-46.

Thomas 1990

Thomas S. Wound management and dressings. London: Pharmaceutical Press, 1990.

\section{Tortora G 1996}

Tortora G, Grabowski S. Principles of Anatomy and Physiology. 8th Edition. New York: Harper Collins College Publicatons, 1996.

\section{Uygur 2004}

Uygur D, Yesildaglar N, Kis S, Sipahi T. Early repair of episiotomy dehiscence. Australian and New Zealand Journal of Obstetrics and Gynaecology 2004;44(3):244-6.

Wagner 2000

Wagner M. Choosing caesarean section. Lancet 2000;356 (9242):1677-80

\section{Williams 2006}

Williams MK, Chames MC. Risk factors for the breakdown of perineal laceration repair after vaginal delivery. American Journal of Obstetrics and Gynecology 2006;195:755-9.

* Indicates the major publication for the study

\section{H ISTORY}

Protocol first published: Issue 2, 2011 


\section{CONTRIBUTIONS OF AUTHORS}

Lynn Dudley (LD) and Christine Kettle (CK) conceived the original idea for the review. LD drafted the clinical sections for the protocol. LD, CK and Khaled MK Ismail (KI) drafted the methodological section of the protocol. All authors were involved in developing the data extraction sheet and agreed the final version of the protocol.

\section{DECLARATIONS OF INTEREST}

The authors of this review (LD, CK and KMKI) are conducting a randomised controlled trial 'The PREVIEW study' (perineal resuturing versus expectant management following vaginal delivery complicated by a dehisced wound) to evaluate the therapeutic effectiveness of resuturing dehisced perineal wounds versus healing by expectant management (secondary intention). The contact person for this review is the Chief Investigator for the PREVIEW study. The study, which will not commence until September 2010, will continue for 18 months and is unlikely to be completed in time for assessment in the initial Cochrane review. In a future update, two independent assessors who are not involved in the PREVIEW trial will conduct independent assessment of eligibility, risk of bias and data extraction for this trial.

Lynn Dudley is a recipient of a Doctoral Nursing Studentship award from the Smith and Nephew Foundation and Research Into Ageing (RIA) which has provided the funding for the trial and to prepare the Cochrane review. The Smith and Nephew Foundation and Research into Ageing do not have any financial interest in the conclusions of this review.

CK and KI run perineal repair workshops both nationally and internationally and have developed an episiotomy and second-degree tear training model with Limbs \& Things, UK. They receive a very small royalty fee for input into the design of the model, which is administered by Keele University Office and Research enterprise and is used as part of their women's health research funds.

\section{SOURCES OF SUPPORT}

\section{Internal sources}

- No sources of support supplied

\section{External sources}

- Lynn Dudley is a recipient of a Doctoral Nursing Studentship award from the Smith and Nephew Foundation and Research Into Ageing (RIA) which have provided the funding to prepare the Cochrane review., UK. 